\title{
Attitudes of patients attending a mental health center towards psychiatric hospitalization
}

\author{
Anastasia Mastrogianni*1, Georgios Papazisis², Georgios Tsenekidis², \\ Constantinos Katsigiannopoulos ${ }^{2}$, Aravella Adamopoulou ${ }^{2}$, \\ Stamatis Donias ${ }^{3}$ and Anastasia Karastergiou ${ }^{1}$
}

Address: ${ }^{1}$ Psychiatric Hospital of Thessaloniki, 196 Langada Street, 56429 Thessaloniki, Greece, ${ }^{2}$ Community Mental Health Center, North-West District, 20 S.Papadopoulou Street, Thessaloniki, Greece and ${ }^{3}$ Community Mental Health Center, Central District, 1 Karaoli \& Dimitriou Street, Thessaloniki, Greece

* Corresponding author

from WPA Thematic Conference. Coercive Treatment in Psychiatry: A Comprehensive Review

Dresden, Germany. 6-8 June 2007

Published: 19 December 2007

BMC Psychiatry 2007, 7(Suppl I):P20 doi:10.1 I86/I47I-244X-7-SI-P20

This abstract is available from: http://www.biomedcentral.com//47I-244X/7/SI/P20

(c) 2007 Mastrogianni et al; licensee BioMed Central Ltd.

\section{Background}

The aim of the study is to explore the attitudes of patients who attend a mental health center towards psychiatric hospitalization and coercive psychiatric treatment.

\section{Methods}

The study is conducted at two mental health centers in Thessaloniki, Greece. Its methodology is similar to the one of the EUNOMIA study, the multi-center research project evaluating coercion in psychiatry. Patients who have been hospitalized at least once in the past are being interviewed. All coercive measures imposed upon them during their last hospitalization (i.e. involuntary admission, restraint, forced medication) as well as their subjective feelings and their attitudes towards the long-term outcome of treatment are recorded. Perceived coercion during admission and hospitalization is also being assessed. Patients comment on their satisfaction with treatment and through a semi-structured interview their knowledge regarding the legal background of coercive psychiatric treatment is being explored.

\section{Results}

Forty patients fulfil the inclusion criteria of the study. The majority are diagnosed with either a psychotic or a mood disorder and they attend the mental health center on a regular basis, receiving supportive psychotherapy individ- ually or in a group. The relation between the degree of coercion experienced by the patients during hospitalization (coercive measures, scores on the MacArthur Perceived Coercion Scale and the Cantril Ladder) and their retrospective evaluation of treatment outcome is explored. Issues such as patients' attitudes towards the pre-conditions of coercive psychiatric treatment and their proposals for best clinical practice are also addressed.

\section{Conclusion}

This study is conducted at outpatient settings by independent researchers, avoiding thus bias and allowing patients to express freely their attitudes towards hospitalization and coercive treatment. Future comparisons with attitudes of inpatients could explore possible differences. 